University of New Hampshire

University of New Hampshire Scholars' Repository

$12-2004$

\title{
National Estimates of Missing Children: Selected Trends, 1988-1999.
}

Heather Hammer

Temple University

David Finkelhor

University of New Hampshire - Main Campus, David.Finkelhor@unh.edu

Andrea J. Sedlack

Westat, Inc.

Lorraine E. Porcellini

Temple University

Follow this and additional works at: https://scholars.unh.edu/ccrc

Part of the Criminology Commons

\section{Recommended Citation}

Heather Hammer, David Finkelhor, Andrea J. Sedlak, \& L. Porcellini. National estimates of missing children: Selected trends, 1988-1999. Juvenile Justice Bulletin - NCJ206179 (pgs. 1-8). Washington, DC: US Government Printing Office.

This Article is brought to you for free and open access by the Research Institutes, Centers and Programs at University of New Hampshire Scholars' Repository. It has been accepted for inclusion in Crimes Against Children Research Center by an authorized administrator of University of New Hampshire Scholars' Repository. For more information, please contact Scholarly.Communication@unh.edu. 
U.S. Department of Justice

Office of Justice Programs

Office of Juvenile Justice and Delinquency Prevention
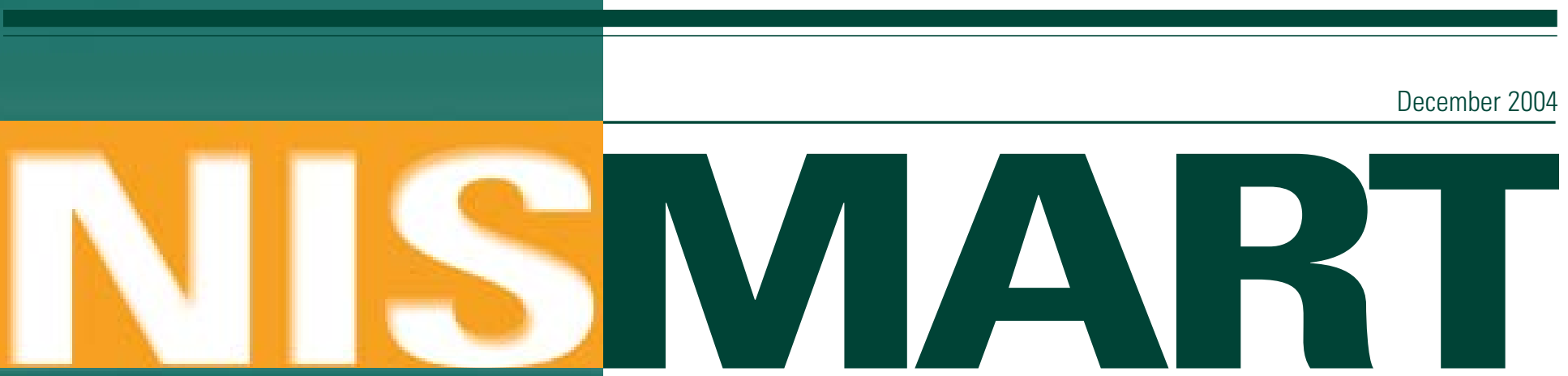

National Incidence Studies of Missing, Abducted, Runaway, and Thrownaway Children

Office of Justice Programs

Partnerships for Safer Communities

www.ojp.usdoj.gov

\section{National Estimates of Missing Children: Selected Trends, 1988-1999}

\author{
Heather Hammer, David Finkelhor, \\ Andrea J. Sedlak, and Lorraine E. Porcellini
}

The words "missing child" call to mind tragic and frightening kidnappings reported in the national news. But a child can be missing for many reasons, and the problem of missing children is far more complex than the headlines suggest. Getting a clear picture of how many children become missing - and why-is an important step in addressing the problem. This series of Bulletins provides that clear picture by summarizing findings from the Second National Incidence Studies of Missing, Abducted, Runaway, and Thrownaway Children (NISMART-2). The series offers national estimates of missing children based on surveys of households, juvenile residential facilities, and law enforcement agencies. It also presents statistical profiles of these children, including their demographic characteristics and the circumstances of their disappearance.

This Bulletin presents results of a special analysis comparing selected findings from NISMART-2 and its predecessor, NISMART-1. The analysis, which is based on household surveys of adult caretakers and covers victims of family abductions, runaways, and children categorized as "lost, injured, or otherwise missing, ${ }^{11}$ highlights trends from 1988 to 1999 and reveals some encouraging news.

\footnotetext{
${ }^{1}$ For methodological reasons, the analysis does not cover nonfamily abductions (including stereotypical kidnappings) and children categorized as "thrownaway." See methodology sidebar on pages $4-7$.
} 


\section{Key Findings}

In the three categories considered (family abductions, runaways, and lost, injured, or otherwise missing children), the analysis finds:

- No evidence of any increase in the incidence of missing children between 1988 and 1999.

Decreases in the incidence rates for some types of episodes of missing children between 1988 and 1999.

\section{Background}

The 1984 Missing Children's Assistance Act (Pub. L. 98-473) requires the Office of Juvenile Justice and Delinquency Prevention (OJJDP) to conduct periodic studies to determine the number of children reported missing and the number recovered in a given year. The first such study, NISMART-1 (Finkelhor, Hotaling, and Sedlak, 1990), provided estimates for 1988 and the second, NISMART-2 (Hammer, Finkelhor, and Sedlak, forthcoming), provided estimates for 1999.

In NISMART-2, the research team changed definitions and methodology to reflect what was learned in NISMART-1. In contrast to the other Bulletins in the NISMART-2 series, this Bulletin uses the original NISMART-1 definitions to permit comparisons between 1999 and 1988. The methodology sidebar on pages 4-7 presents important information for readers to keep in mind as they consider the results that follow.

\section{Results}

This section presents findings for three categories of children: victims of family abductions, runaways, and children categorized as lost, injured, or otherwise missing. The subsections begin with definitions of the cases included in the analysis. In each category, definitions distinguish between "broad scope" and "policy focal" cases-i.e., all cases and those considered to be more serious-as explained below. The findings are summarized in the figure on this page.

\section{Familly Abductions}

Definitions. An episode qualifies as a broad scope family abduction if, in violation of a custody agreement or decree, a family member took a child or failed to return a child at the
Comparison of Incidence Rates for Missing

Children, 1988 (NISMART-1) and 1999

(NISIMART-2)

Rate per 1,000 children ages $0-17$

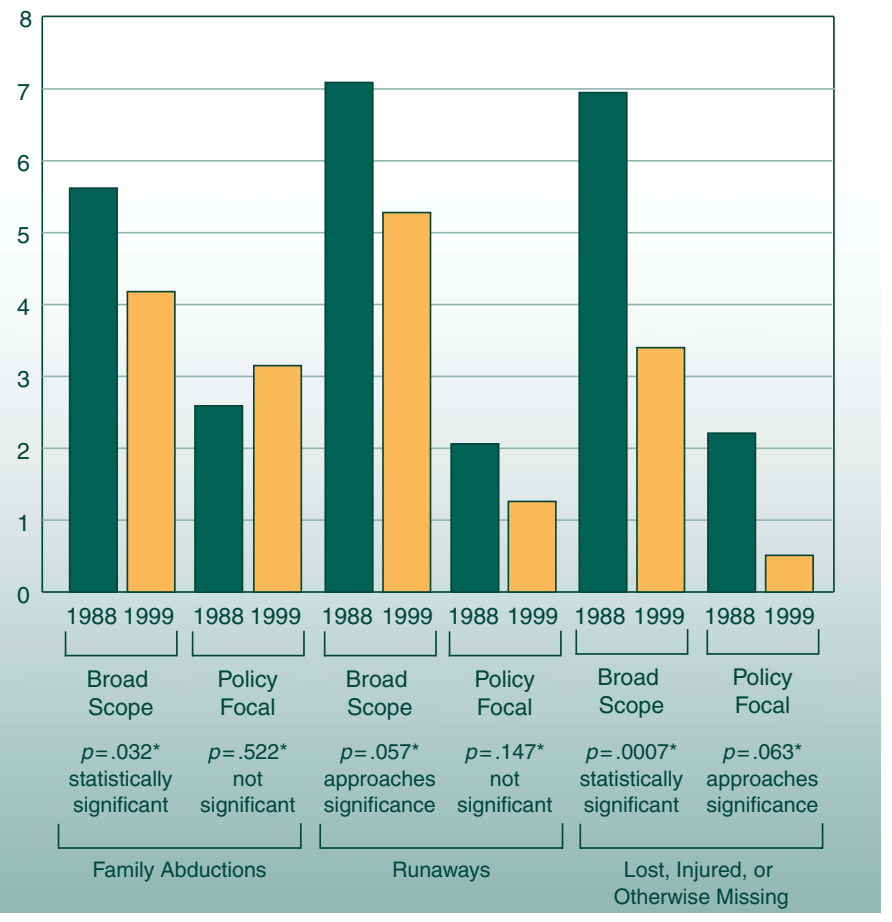

* The $p$ values are the result of tests that measure the extent to which changes between 1988 and 1999 are statistically significant. Two-tailed tests were conducted to detect a change in either direction (increase or decrease), and the conventional level of significance (.05) was used as the cutoff. Thus, $p=.05$ or less indicates that the observed change was statistically significant. Details of the statistical tests, including 95-percent confidence intervals for the rate estimates, will be available in OJJDP's NISMART-2 Household Survey Methodology Technical Report (Hammer and Barr, forthcoming).

end of a legal or agreed-upon visit and the child was kept at least overnight. Policy focal family abductions meet at least one of three additional conditions: (1) the abductor attempted to conceal the taking or whereabouts of the child or to prevent contact with the child, (2) the abductor transported the child out of state, or (3) evidence existed that the abductor intended to keep the child indefinitely or to affect custodial privileges permanently.

The incidence rate for children who experienced broad scope family abductions declined significantly, from 5.62 per 1,000 children in 1988 to 4.18 in 1999. 
The slight increase in the incidence rate for children who were victims of policy focal family abductions, from 2.59 per 1,000 children in 1988 to 3.15 in 1999, is not large enough to be statistically significant-i.e., the difference in rates may simply be due to chance and may not reflect an actual increase in the incidence rate.

One might expect an increase, not a decrease, in the incidence of family abduction victims, given that the population has become more geographically mobile, divorce rates remain high, and parents (particularly fathers) have rising expectations for more equitable custody arrangements. However, countervailing trends may be at work: greater public awareness and avoidance of risks associated with catastrophic conflicts over custody, improved public access to custody dispute resolution services, and court system reforms that make it harder for disgruntled parents to turn to other states for more favorable treatment.

Such factors may explain the decline in the incidence rate for children who experienced broad scope family abductions. The lack of decline in the incidence rate for children who experienced more serious policy focal family abductions may mean that these improvements have had less impact on the most acrimonious, combative custody situations in which noncustodial parents resort to extreme unilateral actions.

At least one demographic change between 1988 and 1999 may have affected family abduction estimates: a decline in the number of children who live with both biological parents. During that period, the U.S. Census Bureau's Current Population Surveys (conducted in March of each year) show a 4.6-percent decline in the number of children living with both parents, and NISMART caretaker respondents who were biological parents of the child in question declined 4 percent between NISMART-1 and NISMART-2. In NISMART-1, 9 out of 10 respondents were parents, and the rest were primarily grandparents, aunts, and uncles (Finkelhor, Hotaling, and Sedlak, 1992); in NISMART-2, fewer (86 percent) were biological parents, more ( 4 percent) were stepparents, and 6 percent (about the same as in NISMART-1) were grandparents, aunts, and uncles.

The increase in the percentage of NISMART caretakers who are stepparents may be especially relevant to the decline in the incidence rate for children who experienced broad scope family abductions. Stepparents may be less aware of, or less likely to report, less serious family abductions, which tend to involve conflicts between biological parents.

\section{Runaways}

Definitions. An episode qualifies as a broad scope runaway if it meets one of the following criteria: (1) a child left home without permission and was away at least one night; (2) a child made a statement or left a note indicating intent to run away and then stayed away at least overnight; (3) a child age 15 or older was away, chose not to come home when expected, and stayed away at least two nights; or (4) a child age 14 or younger was away, chose not to come home when expected, and stayed away at least one night. Policy focal runaway episodes meet the additional condition that the child was without a familiar and secure place to stay for at least one night.

The incidence rate for broad scope runaways declined from 7.09 per 1,000 children in 1988 to 5.28 in 1999. This decrease approaches statistical significance, meaning that it probably, but not conclusively, indicates an actual decline.

The decrease in the incidence rate for policy focal runaways, from 2.06 per 1,000 children in 1988 to 1.26 in 1999, is not large enough to be statistically significant. The difference in rates may simply be due to chance and may not reflect an actual decrease in these cases.

The likely decline in the incidence rate for broad scope runaways and the possible decline in the incidence rate for policy focal runaways could have a variety of explanations. As context, it is important to note that most runaway episodes (as defined in NISMART) are brief, lasting no longer than a day or two. These episodes often involve children doing things they think their parents may disapprove of, such as traveling to a distant party or event, or spending time with a boyfriend or girlfriend.

Between 1988 and 1999, caretakers may have begun to give teenagers greater independence or may have become less able to establish limits in light of competing 


\section{Methodology}

This section presents background information on NISMART-1 and NISMART-2 that readers should take into account when considering the findings presented in this Bulletin. It discusses the reasons for excluding certain categories of missing children from the analysis, examines differences in the two surveys' methodology and definitions, presents brief technical notes, and compares the NISMART-1 and NISMART-2 household samples.

\section{Exclusions}

Nonfamily abductions. Because NISMART-1 and NISMART-2 used different methods to estimate the number of children who are victims of nonfamily abductions, the findings from the two surveys are not comparable. Missing children represent a small fraction of the total number of children in the population, and very serious cases, such as children who are victims of stereotypical kidnappings by strangers, are rare. In NISMART-1, the household survey did not yield enough cases to permit calculation of a national estimate of nonfamily abductions or stereotypical kidnappings (Finkelhor, Hotaling, and Sedlak, 1990); instead, the estimate was derived from a police records study. The NISMART-2 design sought to improve estimates by combining two methods: a household survey to estimate less serious nonfamily abductions and a survey of law enforcement agencies to ensure an accurate estimate of stereotypical kidnapping victims.
Thrownaway children. Thrownaway children are those whom an adult in the household has asked or told to leave home. The NISMART-2 Household Survey of Adult Caretakers yielded only seven thrownaway cases - too few to support an estimate. Findings from the NISMART-2 Household Survey of Youth and the NISMART-1 study of returned runaways (Finkelhor, Hotaling, and Sedlak, 1990) suggest that the small number of NISMART-2 thrownaways may not reflect an actual decline between 1988 and 1999. It is more likely that caretakers are increasingly reluctant to report thrownaway episodes, less likely to recall such episodes in interviews, or more inclined to remember and report the runaway aspects of episodes that possibly involved both runaway and thrownaway elements.

\section{Differences in Methodology and Definitions}

Because a key objective of NISMART is to detect historical changes, NISMART-2 designers retained many core elements from NISMART-1. On the other hand, designers also sought to make improvements based on experience from NISMART-1, and these improvements involved several changes in methodology and definitions. These changes mean that data and findings from the two surveys should not be compared directly.

- Virtually all of the key questions asked in NISMART-1 to determine whether an episode should be counted were asked again in NISMART-2. However, questionnaire demands on caretakers' time. This could mean that fewer children who are away from home meet the runaway definition of being away without permission.

Another possible factor is the increasing availability of cell phones and other modes of communication. Children who have taken liberties may be more likely to negotiate an agreement with their caretakers, precluding a sense of alarm or violation of permission.

In addition, running away may hold less allure as children become more aware of the dangers involved. The Internet may now serve as a substitute for hanging out with friends, taking risks, and having adventures. The decline in running away is also consistent with improvements in child and youth well-being during the 1990s (Foundation for Child Development, 2004). These improvements include declines in child poverty (Federal Interagency Forum, 2000), teenage drinking (Johnston, O'Malley, and Bachman, 2000) and pregnancy (Federal Interagency Forum, 2000), youth violence and victimization (U.S. Bureau of Justice Statistics, 2000), and child abuse and neglect, including sexual abuse (Jones and Finkelhor, 2001; Finkelhor and Jones, 2004).

\section{Lost, Injured, or Otherwise Missing Children}

Definitions. An episode qualifies as broad scope lost, injured, or otherwise missing if it meets one of the following criteria: (1) a child disappeared from home or from parental supervision and could not be located for varying amounts of time depending on age-any amount of time (ages 0-2), 2 hours (3-4), 3 hours (5-6), 4 hours (7-10), 8 hours (11-13), overnight (14-17)—or, for a child of any age with a serious or permanent physical or mental disability or impairment or life threatening medical condition, for 1 hour; (2) a child who was out with parental permission failed to return, could not be located, and was gone at least overnight; or (3) a child who was out with parental permission failed to return or make contact with the parent for at least an 
format and content changed. For example, not all questions were replicated verbatim and, in some instances, the designers changed question format from openended to close-ended, changed question order, collapsed sequences of questions into a single question, or partitioned a single question into a sequence.

- Other analyses in this series of Bulletins focus entirely on NISMART-2 and use that survey's definitions to calculate estimates. However, to permit comparisons with NISMART-1 data in this Bulletin, researchers calculated NISMART-2 estimates according to the original NISMART-1 definitions. Because the extent to which changes in questionnaire format and content may have influenced responses is not clear, these estimates are close approximations to (but not strict replications of) the original NISMART-1 definitions.

- NISMART-2 included a household survey of youth. NISMART-1 did not. Therefore, comparisons between the two years must be limited to the adult caretaker household surveys conducted in both years.

\section{Technical Notes: Rates, Weighting, and Sampling Errors}

According to the U.S. Census Bureau, the population ages 0-17 increased from just over 63 million in 1988 to almost 72 million in 1999. Because of this change, the analysis in this Bulletin standardizes the incidence estimates for
NISMART-1 and NISMART-2, reports the estimates as incidence rates of missing children per 1,000 children ages 0-17 in the population, and measures change in these rates over time.

Standardizing the survey data involved weighting the data to reflect the census-based population ages $0-17$ at the time of each survey. All comparisons between the two surveys take into account the fact that the estimates are based on samples and, as a result, sampling error affects their precision. This sampling error is reflected in the confidence interval around each estimated rate-i.e., a range within which the true number should fall 95 percent of the time when a study like this one is conducted. Details of the NISMART-1 weighting procedures and variance estimation are available in NISMART Household Survey Methodology (Sedlak, Mohadjer, and Hudock, 1990). Similar details for NISMART-2 will be available in OJJDP's NISMART-2 Household Survey MethodologyTechnical Report (Hammer and Barr, forthcoming).

\section{Comparing the NISMART-1 and NISMART-2 Household Samples}

A primary challenge in designing the NISMART-2 household survey was to have a large enough sample to support an estimate of the number of children who were victims of nonfamily abductions. Thus, NISMART-2 attempted to reach a much larger household sample of adult primary caretakers than was reached in NISMART-1. The table on page 7 hour after return, or contact was expected because the child suffered harm or an injury that required medical attention. Policy focal lost, injured, or otherwise missing episodes meet the additional condition that the police were contacted to help locate the child.

- The incidence rate for children who experienced broad scope lost, injured, otherwise missing episodes decreased significantly, from 6.95 per 1,000 children in 1988 to 3.40 in 1999.

The incidence rate for children who experienced policy focal lost, injured, otherwise missing episodes declined from 2.21 per 1,000 children in 1988 to 0.51 in 1999. This decrease approaches statistical significance, meaning that it probably, but not conclusively, indicates an actual decline.

These findings suggest a clear decline in the incidence rate for children who experienced broad scope lost, injured, or otherwise missing episodes and a possible decline in the incidence rate for children who experienced episodes classified as policy focal because they triggered a call to police to help locate the missing child. Most commonly, lost, injured, or otherwise missing episodes involve children who are delayed coming home or contacting their parents because of weather, travel delays (such as car breakdowns), and other extenuating circumstances, and children who are perceived as missing because of a miscommunication about their plans or intentions.

Once again, a factor in the observed decline could be improvements in communication technology, such as the proliferation of cell phones. However, it is also possible that the change could arise from methodological differences between NISMART-1 and NISMART-2.

\section{Summary}

This analysis examines trends in the incidence of children who are family abduction victims, runaways, and lost, injured, or otherwise missing, based on NISMART data for 1988 and 1999. It considers children who experienced a broad scope (i.e., any) incident and those who experienced a policy focal (more serious) incident. 
presents detailed sample comparisons for NISMART-1 and NISMART-2 and reveals significant differences between the two surveys. Although NISMART-2 contacted nearly 2.5 times as many households as NISMART-1, the numbers of interviews completed and children represented were not proportionately larger.

Of the 85,522 households contacted in NISMART-2, it was possible to screen 85 percent for the presence of children, yielding 20,170 eligible households with children. Of these, 16,111 households (representing 31,787 children) yielded completed adult interviews, for a response rate of 61 percent. In NISMART-1, 11,617 eligible households yielded 10,367 completed interviews (representing 20,138 children), for a response rate of 78 percent.*

The recruitment statistics in the table show that the NISMART-2 sample yielded proportionately fewer contacts with households, fewer eligible households with children, fewer completed interviews among eligible households, and more telephone numbers with unknown eligibility. Only the percent of ineligible telephone numbers is similar between the two surveys.

The outcome rates in the table, which are based on American Association for Public Opinion Research standard definitions (AAPOR, 2004), mirror the recruitment

*Response rates for NISMART-1 and NISMART-2 have been computed with standard definition RR4 (AAPOR, 2004) to facilitate this comparison. statistics. NISMART-2 had a significantly lower contact rate (the proportion of all cases in which the survey reached some responsible member of the household) and cooperation rate (the proportion of completed interviews among eligible households) and a somewhat higher refusal rate (the proportion of all cases in which a household or respondent refuses to do an interview or breaks off an interview among all potentially eligible households). These outcomes yielded a lower response rate (the proportion of completed interviews among all eligible households in the sample) for NISMART-2. This is consistent with the increasing prevalence of nonresponse (including noncontact and refusals) noted by other researchers in general population surveys, particularly in random-digit dial surveys like NISMART (Groves and Couper, 1998; Hox and De Leeuw, 1994; Harris-Kojetin and Tucker, 1999; Steeh et al., 2001).

The recruitment and outcome differences between NISMART-1 and NISMART-2 may stem from their use of different methodologies to sample and screen phone numbers. The differences may also reflect an increased reluctance of respondents to report the presence of children living in the household, or possibly a larger proportion of eligible households (i.e., households with children) "hidden" in phone numbers with unknown eligibility.

A discussion of these differences and their potential implications will be available in OJJDP's NISMART-2 Household Survey Methodology Technical Report (Hammer and Barr, forthcoming).
The analysis offers evidence of statistically significant declines in incidence rates for children who experienced broad scope family abduction episodes and lost, injured, or otherwise missing episodes, plus some evidence of declines in incidence rates for children who experienced broad scope runaway episodes and policy focal lost, injured, otherwise missing episodes. Observed changes in incidence rates for children who experienced policy focal family abduction episodes and policy focal runaway episodes are not statistically significant.

The most important finding is the absence of increases in any of these problems. This finding is consistent with growing evidence of improvements in child and youth well-being during the 1990s (Foundation for Child Development, 2004; Federal Interagency Forum, 2000; Johnston, O'Malley, and Bachman, 2000; U.S. Bureau of Justice Statistics, 2000; Jones and Finkelhor, 2001; Finkelhor and Jones, 2004).

Such evidence suggests that the environment affecting children-possibly the economic resources available for their care, the quality of family life and parental attention, availability of social and medical services, and community safety and cohesion-has not worsened and may have improved. It is also possible that the allure of running away may have dissipated as parents have given children greater freedom and independence. The proliferation of cell phones and other modes of communication has made it easier to locate children and clear up misunderstandings, which may help to explain the decline in lost, injured, or otherwise missing children.

Although the findings reported in this Bulletin are encouraging, they are no cause for complacency. The NISMART estimates for 1999 also reveal large numbers of children and youth still caught up in circumstances of crisis and vulnerability. The family and community problems these statistics reflect are unlikely to disappear anytime soon.

Finally, methodological differences between the 1988 and 1999 NISMART surveys (see sidebar on pages 4-7) may explain some of the change (and lack of change) reported in this Bulletin. Further analysis is required to 
Sample Statistics for NISIMART-1 and NISIMART-2 Household Surveys

Sample Statistic Category

\section{Recruitment}

Telephone numbers called

Households contacted

Numbers with unknown eligibility

Ineligible numbers

Households screened for children

Screened households with children

Completed interviews with adult caretakers

Children in households where adults completed interviews

Outcome Rates ${ }^{d}$

Contact rate

Cooperation rate

Refusal rate

Response rate

Note: All percents are rounded to the nearest whole number.

a Percent computed from total households contacted.

b Percent computed from households screened for children.
NISMART-1

Number

60,000

34,820

7,731

40,652

30,268

11,617

10,367

20,138

Per

\section{Percent}

100

58

13

68

$87^{a}$

$38^{\mathrm{b}}$

$89^{\circ}$

Percent

87

89

11

78
NISMART-2

$\begin{array}{rc}\text { Number } & \text { Percent } \\ 188,477 & 100 \\ 85,522 & 45 \\ 44,318 & 23 \\ 123,989 & 66 \\ 73,055 & 85^{\mathrm{a}} \\ 20,170 & 28^{\mathrm{b}} \\ 16,111 & 80^{\mathrm{c}} \\ 31,787 & \end{array}$

Percent

77

80

15

61 reach definitive conclusions about the impact of these differences.

\section{Conclusion}

The period between 1988 and 1999 saw significant mobilization on behalf of missing children. Law enforcement officers received special training, and public awareness grew as a result of media coverage and educational programs disseminated to schools and families. Have these efforts made a difference? What else should be done? The kinds of data collected thus far are of limited use in answering these questions. Data gathered more frequently and more locally could provide a tool for public policy analysis that might lead to even greater effectiveness in combating the problem of missing children.

\section{References}

American Association for Public Opinion Research (AAPOR). 2004. Standard Definitions: Final Dispositions of Case Codes and Outcome Rates for Surveys. 3rd edition. Lenexa, KS:

AAPOR.

Federal Interagency Forum on Child and Family Statistics. 2000. America's Children: Key National Indicators of WellBeing, 2000. Washington, DC: U.S. Government Printing Office.

Finkelhor, D., Hotaling, G., and Sedlak, A. 1990. Missing, Abducted, Runaway, and Thrownaway Children in America. First Report: Numbers and Characteristics-National Incidence
Studies. Washington, DC: U.S. Department of Justice, Office of Justice Programs, Office of Juvenile Justice and Delinquency Prevention.

Finkelhor, D., Hotaling, G., and Sedlak, A. 1992. The abduction of children by strangers and nonfamily members: Estimating the incidence using multiple methods. Journal of Interpersonal Violence 7(2):226-243.

Finkelhor, D., and Jones, L.M. 2004. Explanations for the Decline in Child Sexual Abuse Cases. Bulletin. Washington, DC: U.S. Department of Justice, Office of Justice Programs, Office of Juvenile Justice and Delinquency Prevention.

Foundation for Child Development. 2004. The Foundation for Child Development Index of Child Well-Being (CWI), 1975-2002. Durham, NC: Duke University, Foundation for Child Development.

Groves, R.M., and Couper, M.P. 1998. Nonresponse in Household Interview Surveys. New York, NY: John Wiley and Sons, Inc.

Hammer, H., and Barr, M.K. Forthcoming. NISMART-2 Household Survey Methodology Technical Report.

Hammer, H., Finkelhor, D., and Sedlak, A. Forthcoming. Missing, Abducted, Runaway, and Thrownaway Children in America. Final Report of Estimates From the Second National Incidence Studies, NISMART-2. Washington, DC: U.S. Department of Justice, Office of Justice Programs, Office of Juvenile Justice and Delinquency Prevention. 
Office of Justice Programs

Washington, DC 20531

Official Business

Penalty for Private Use $\$ 300$

Harris-Kojetin, B., and Tucker, C. 1999. Exploring the relation of economic and political conditions with refusal rates to a government survey. Journal of Official Statistics 15(2):67-184.

Hox, J.J., and De Leeuw, E.D. 1994. A comparison of nonresponse in mail, telephone, and face-to-face surveys. Applying multilevel modeling to meta-analysis. Quality and Quantity 28:329-344.

Johnston, L.D., O'Malley, P.M., and Bachman, J.G. 2000. Monitoring the Future. National Results on Adolescent Drug Use: Overview of Key Findings 1999. Washington, DC: U.S. Department of Health and Human Services.

Jones, L., and Finkelhor, D. 2001. The Decline in Sexual Abuse Cases. Bulletin. Washington, DC: U.S. Department of Justice, Office of Justice Programs, Office of Juvenile Justice and Delinquency Prevention.

Sedlak, A.J., Mohadjer, L., and Hudock, V. 1990. NISMART Household Survey Methodology. Washington, DC: U.S. Government Printing Office.

Steeh, C., Kirgis, N., Cannon, B., and DeWitt, J. 2001. Are they really as bad as they seem? Nonresponse rates at the end of the twentieth century. Journal of Official Statistics 17(2):227-247.

U.S. Bureau of Justice Statistics. 2000. National Crime Victimization Survey trends, 1973-98: Adjusted victimization rates. Retrieved August 13, 2000, from the Web: www.ojp.usdoj.gov/bjs.
This Bulletin was prepared under grant number 95-MC-CX-K004 from the Office of Juvenile Justice and Delinquency Prevention, U.S. Department of Justice, to Temple University.

Points of view or opinions expressed in this document are those of the authors and do not necessarily represent the official position or policies of OJJDP or the U.S. Department of Justice.

The Office of Juvenile Justice and Delinquency Prevention is a component of the Office of Justice Programs, which also includes the Bureau of Justice Assistance, the Bureau of Justice Statistics, the National Institute of Justice, and the Office for Victims of Crime.

\section{Acknowledgments}

Heather Hammer, Ph.D., is a Senior Study Director at the Temple University Institute for Survey Research, Philadelphia, PA, and Principal Investigator of NISMART-2. David Finkelhor, Ph.D., is Professor of Sociology and Director of the Crimes against Children Research Center, University of New Hampshire; and Advisor to NISMART-2. Andrea J. Sedlak, Ph.D., is Associate Director of Human Services Research at Westat, Inc.; Project Director of the NISMART-2 Unified Estimate, Juvenile Facilities Study, and Law Enforcement Study; and Advisor to the NISMART-2 Household Survey. Lorraine E. Porcellini, B.A., is Study Direction/Sampling Coordinator at the Temple University Institute for Survey Research.

The authors extend their appreciation to the many individuals who contributed to this Bulletin, to OJJDP NISMART-2 Program Manager Barbara Allen-Hagen, and to the NISMART survey respondents. 